\title{
Implementation of a Solution to the Conjugacy Problem in Thompson's Group F
}

\author{
James Belk, Bard College, belk@bard.edu, \\ Nabil Hossain, Bard College, nh1682@bard.edu \\ Francesco Matucci, Université Paris-Sud, \\ francesco.matucci@math.u-psud.fr \\ Robert W. McGrail, Bard College, mcgrail@bard.edu
}

\begin{abstract}
We present an efficient implementation of the solution to the conjugacy problem in Thompson's group $F$. This algorithm checks for conjugacy by constructing and comparing directed graphs called strand diagrams. We provide a description of our solution algorithm, including the data structure that represents strand diagrams and supports simplifications.
\end{abstract}

\section{Thompson's Group F and Strand Diagrams}

The elements of Thompson's Group $F[3]$ are piecewise, linear homeomorphisms of the interval $[0,1]$ such that each piece has slope that is a power of 2 and, furthermore, the breakpoints between pieces take place at dyadic rational coordinates. The group operation is simply function composition. In a group, the conjugacy problem is the problem of determining whether any two elements are conjugate. The conjugacy problem is not solvable in general [5], but is solvable in certain cases.

A strand diagram [2] is a finite acyclic digraph embedded on the unit square. The digraph has a source along the top edge of the square and a sink along the bottom edge. Any internal vertex is either a merge or a split (Figure 1). Elements of Thompson's Group $F$ can be translated to strand diagrams. Each element in a generating set corresponds to a particular strand diagram. A composition of such elements is represented by a concatenation of the associated strand diagrams.
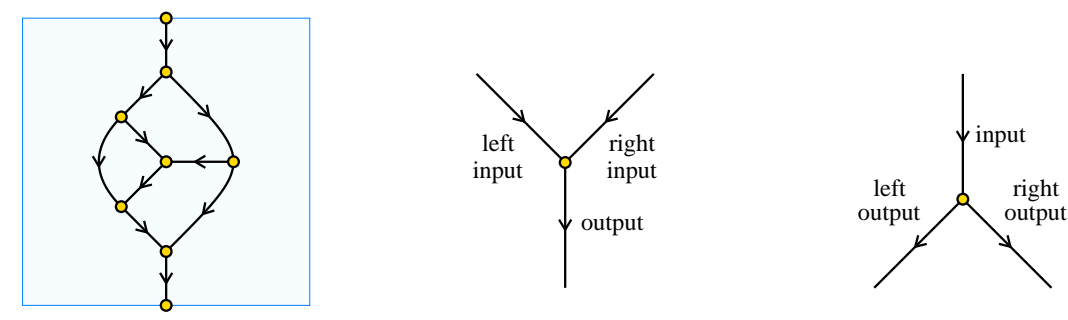

Figure 1: A strand diagram, a merge, and a split (image taken from [2]). 


\section{Algorithm for the Conjugacy Problem in $F$}

The algorithm to determine whether two strand diagrams inhabit the same conjugacy class proceeds as follows. First, we convert the strand diagrams to annular strand diagrams. This is achieved by a process called closing, in which sources are identified with sinks. Next, the annular strand diagrams are reduced using a graphical rewriting system that is both confluent, terminating, and respects conjugacy [1]. Furthermore, any two connected and reduced annular strand diagrams $s_{1}$ and $s_{2}$ can be encoded into two planar graphs $g_{1}$ and $g_{2}$ respectively such that $s_{1}$ and $s_{2}$ represent conjugate elements if and only if $g_{1}$ and $g_{2}$ are isomorphic. Hence the problem reduces to checking whether two simplified planar graphs are isomorphic. Moreover, this enterprise can be carried out in linear time given a linear time planar-graph-isomorphism checker [4].

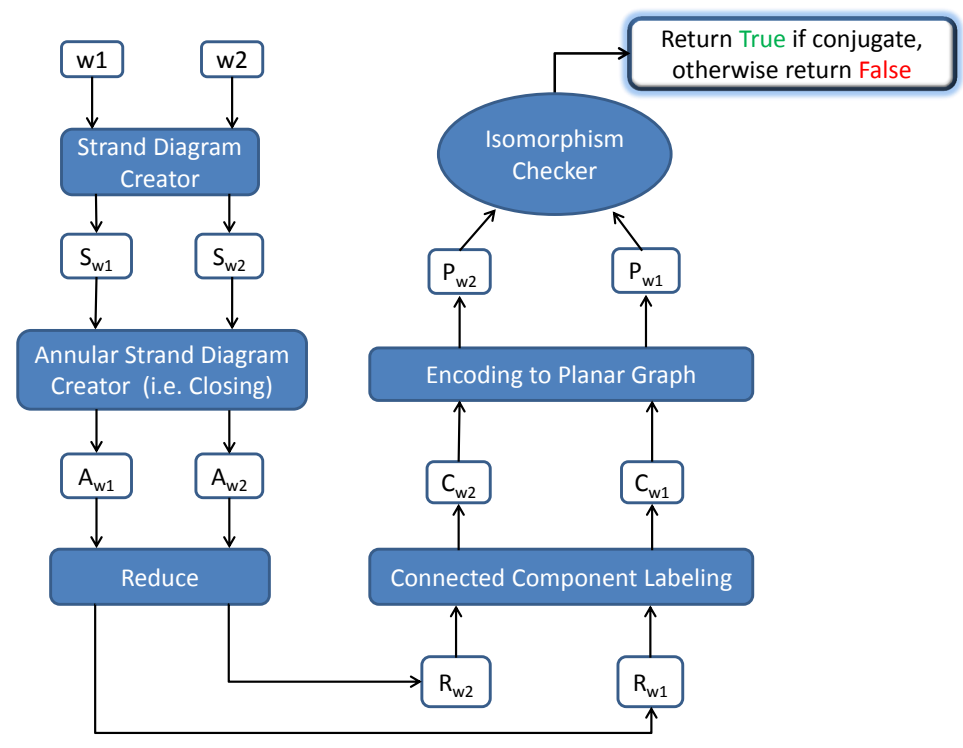

Figure 2: Algorithm Flowchart

\section{References}

[1] F. Baader and T. Nipkow. Term Rewriting and All That. Cambridge University Press, 1999.

[2] J. Belk and F. Matucci. Conjugacy and dynamics in Thompson's groups. Preprint, 2013.

[3] J. W. Canon, W. J. Floyd, and W. R. Parry. Introductory notes on Richard Thompson's groups. Enseignement Mathématique, 42: 215-256, 1996.

[4] J. E. Hopcroft and J. K. Wong. Linear time algorithm for isomorphism of planar graphs (preliminary report). Proceedings of the Sixth Annual ACM symposium on Theory of Computing, $172-184,1974$.

[5] P. S. Novikov. Unsolvability of the conjugacy problem in the theory of groups. Izv. Akad. Nauk SSSR. Ser. Mat, 18: 485-524,1954. 